\title{
Influence of the Fractal Geometry on the Mechanical Resistance of Cantilever Beams Designed through Topology Optimization
}

\author{
Pablo Pavón-Domínguez *D, Guillermo Portillo-García, Alejandro Rincón-Casado \\ and Lucía Rodríguez-Parada (D)
}

check for

updates

Citation: Pavón-Domínguez, P.; Portillo-García, G.; Rincón-Casado,

A.; Rodríguez-Parada, L. Influence of

the Fractal Geometry on the

Mechanical Resistance of Cantilever Beams Designed through Topology Optimization. Appl. Sci. 2021, 11, 10554. https://doi.org/10.3390/ app112210554

Academic Editor: Francisco Cavas Martínez

Received: 27 September 2021 Accepted: 4 November 2021 Published: 9 November 2021

Publisher's Note: MDPI stays neutral with regard to jurisdictional claims in published maps and institutional affiliations.

Copyright: (c) 2021 by the authors. Licensee MDPI, Basel, Switzerland. This article is an open access article distributed under the terms and conditions of the Creative Commons Attribution (CC BY) license (https:// creativecommons.org/licenses/by/ $4.0 /)$.
Department of Mechanical Engineering and Industrial Design, College of Engineering, University of Cadiz, Avenida de la Universidad de Cádiz, 11519 Puerto Real, Cádiz, Spain; guiporgar@gmail.com (G.P.-G.); alejandro.rincon@uca.es (A.R.-C.); lucia.rodriguez@uca.es (L.R.-P.)

* Correspondence: pablo.pavon@uca.es

\begin{abstract}
In this work, the complex geometry of beams obtained from topology optimization is characterized through the fractal dimension $\left(F_{D}\right)$. The fractal dimension is employed as an efficiency measure of the mass distribution in the beams, that is, the capacity of the optimized solutions to be efficiently distributed in the design space. Furthermore, the possible relationships between the fractal dimension and beams' mechanical properties are explored. First, a set of theoretical beams are studied based on their well-known fractal dimension. A 3D fractal called Menger sponge is reproduced on a Michell's beam (cantilever with a single force applied at the end). The programming codes that generate those beams are created in Matlab software, as are the algorithms for estimating the fractal dimension (box-counting method). Subsequently, identical beams are modelled in the software Inspire in order to apply the topology optimization and determine the mechanical parameters from the static analysis. Results indicate that the fractal dimension is affected by the design geometry and proposed optimized solutions. In addition, several relationships among fractal dimension and some mechanical resistance parameters could be established. The obtained relations depended on the objectives that were initially defined in the topology optimization.
\end{abstract}

Keywords: fractal dimension; topology optimization; static analysis; box-counting; Michell's beam

\section{Introduction}

Fractal geometry [1] is one of the most established theories for characterizing complex geometries. This geometry, also called geometry of nature, makes it possible to describe complex shapes and relate them to mechanical, rheological and morphological properties [2]. Optimal structures have a grillage pattern at both macro- and microscales, such as natural (e.g., bones) and artificial structures [3]. Moreover, biomimetics is gaining relevance as a tool to solve problems in engineering by means of the emulation and application of natural geometries and shapes [4,5]. Fractal geometry is a suitable candidate for generating structures based on natural shapes due to its ability to construct repetitive and hierarchical self-similar patterns [6].

Because natural shapes are related to mechanical, acoustic and electrical properties in nature [7], it might be interesting to know if there is a relationship between fractal geometry and mechanical properties in human-made objects. However, the literature about associations between objects constructed or generated by fractal geometries and their physical properties is scarce [7]. Some works have explored the potential of generating fractal structures for optimizing physical properties, for example in branching channel networks of internal cooling passages [8], fractal-like geometries for microchannel heat sinks [9] and bionic optimizations for enhancing latent heat thermal energy storage performances [10]. In ref. [11], the influence of fractal dimension on the porosity, permeability and thermal conductivity of porous fibrous materials was studied, showing interesting results when fractal dimension was included as a comparative parameter. 
On the other hand, topology optimization is a design method to obtain optimized structures. Topology optimization aims to determine the optimal distribution of a material within a design domain by considering both a set of boundary conditions and performance objectives [12]. This procedure arises as a solution for defining geometries in order to minimize the cost of the product, while maintaining the constraints and fitting the boundary conditions [13]. Topology optimization is based on iterative mathematical algorithms for reaching a design goal. In general, this process aims to minimize the amount of material for designing and manufacturing a product, while meeting a set of restrictions (stresses, limitation of displacement, stiffness, maximum deviation, etc.). Generally, topology optimization methods employ finite element methods (FEMs) and are common add-ons in computeraided design programs such as Inspire, Solidworks, Fusion 360, etc. The main advantage of product design based on topology optimization is to obtain pieces with reduced weight and volume without compromising their mechanical specifications. However, the complex geometry of these solutions can make them difficult to manufacture. This disadvantage has been overcome thanks to advances in additive manufacturing (e.g., 3D printing).

Inspired on the so-called Michell's beam [14], this work is conducted using a cantilever beam as a case study. This is the first mention of structural topology optimization and dates back to 1904 [15]. It is based on a geometrically unconstrained problem, in which the mechanical compliance of the optimized object is optimal with respect to stress [16]. The pioneer topology optimization developed by [14] was based on truss structures, and later extended to grillages (beam systems) in $[17,18]$. In this context, the application of fractal geometry for generating tensegrity structures has been explored by means of self-similar subdivisions of finite or infinite basic modules $[19,20]$. This makes it possible to generate tensegrity fractals that optimize the values of suitable complexity parameters under mechanical performance criteria [21]. Topology optimization has also focused on other objectives, such as the minimization of the dynamic components in the frequency range of vibration [22]. After reviewing the latest works on two-dimensional and three-dimensional topology optimization, a trend to study weakly coupled fluid-structure systems is observed [23], providing topology optimization models for different engineering applications and taking into account the optimization of the volume. Currently, topology optimization solutions provide information about the percentage of maintained material with respect to the original material. However, different geometric configurations can be obtained with equal percentage of material. Fractal dimension is a descriptive parameter about the way in which a material fills the space, so it could be useful for evaluating how efficient the optimized solution is from a geometric approach. However, few works consider the fractal dimension as an additional parameter for the objective function of topology optimizations. Thus, we hypothesize that fractal dimension might be a complementary parameter of the maintained mass, adding useful information about the geometrical efficiency of the optimized solution.

This work is intended as an initial exploration for the emerging use of fractal theory in structural analysis. To our best knowledge, this paper represents the first exploration of the fractal dimension $\left(F_{D}\right)$ and its relation to mechanical strength properties. Therefore, we hypothesize that some fractal properties (e.g., fractal dimension [14]) can be compared with topology results such as mechanical strength and mass fraction of the optimized part. In this way, we intend to relate the geometric properties of the organic shapes obtained by topology optimization with their mechanical strength properties. On the one hand, the solutions obtained by topology optimization evoke organic shapes, and on the other, the geometry of these optimized parts is distributed in space to minimize the amount of material without compromising their mechanical strength. For both reasons, this work suggests that the optimized parts could show an efficient spatial distribution of their geometry, which can be related through the fractal dimension. First, fractal dimension is used as a measure of the efficiency in the geometric distribution of pieces obtained through topology optimization. Second, these solutions are assessed and compared with mechanical resistance parameters, as well as with their mass and volume. The objectives 
of the work are to (i) demonstrate that the geometry of the pieces obtained from topology optimization can be characterized by a fractal dimension, and (ii) evaluate whether there is a relationship between the fractal dimension and the mechanical resistance parameters.

\section{Materials and Methods}

\subsection{Fractal Dimension}

Fractal dimension $\left(F_{D}\right)$ is a value that characterizes fractal objects [24] by assigning them a non-integer value between two topological dimensions ( 0 in the case of a point, 1 for a line, 2 for a plane and 3 for a volume). The so-called Hausdorff-Besicovitch dimension $\left(D_{H B}\right)$ is applied for the analytical determination of an object's fractal dimension:

$$
D_{H B}=\frac{\ln [L(\delta)]}{\ln [\delta]}
$$

where $L[\delta]$ represents the number of repetitions of the unit and $\delta$ is the observation scale. Equation (1) is used when fractal objects have an infinite detail (i.e., they are theoretical objects such as mathematical fractals). By contrast, real objects are limited by a finite range of scales, and thus the iterations for generating them are finite. Therefore, estimation procedures are frequently used for computing the fractal dimension of real objects. The box-counting method is one of the most widely used procedures due to its simplicity and the reliability of the results obtained. Hence, box-counting is chosen in this work for calculating the fractal dimension of the beams.

Box-counting is one of the so-called counting methods [25]. In these methods, magnitudes are quantified by using successive partitions of different sizes and by counting the amount of portions of the object that belong to the aforementioned partitions. In the case of box-counting, the object is successively subdivided into boxes of equal size, varying the size of these boxes so that they become smaller and smaller. Next, the number of boxes containing portions of the fractal object are counted. When the relationship between the box size $(\delta)$ and the number of boxes containing the object $L(\delta)$ follow a power law, fractal properties are evinced (Figure 1a). When a double logarithmic plot represents the aforementioned relationship, the power law is transformed into a linear function. According to Equation (1), the fractal dimension can be estimated as the quotient between $\ln [L(\delta)]$ versus $\ln [\delta]$ - that is, the slope of the linear fit (Figure 1b). As an example, the estimation of the fractal dimension of the Sierpinski carpet is shown in Figure 1. The fractal dimension of the Sierpinski carpet can be analytically calculated by means the quotient between $\ln (8)$ versus $\ln (3)$, resulting around 1.893 (https:/ / en.wikipedia.org/wiki/Sierpi\%C5\%84ski_carpet) [accessed on 3 September 2021]. This fractal dimension between 1 and 2 indicates that the Sierpinski carpet is a fractal object with a dimension greater than a line, but less than a plane.

The third iteration of the Sierpinski carpet (Figure 2) is employed for this example, obtaining an estimated fractal dimension of around 1.751. The estimated fractal dimension by means of the box-counting method is close to the analytical result. The difference is due to the use of a finite fractal (third iteration). The estimated fractal dimension should become closer to the theoretical value as the iterations increase.

\subsection{Beam Based on the Fractal Geometry of the Menger Sponge}

In this work, the exploration of the fractal geometry in beams is based on the Menger sponge. The Menger sponge is a three-dimensional generalization of the Sierpinski carpet, and was developed in 1926 by Karl Menger.

Let us consider a regular hexahedron. The Menger sponge is obtained by subdividing each face of the hexahedron into nine identical squares. Thus, 27 identical regular sub-hexahedra are obtained. Subsequently, the 7 central sub-hexahedra are subtracted, maintaining 20 regular sub-hexahedra (https:/ / en.wikipedia.org/wiki/Menger_sponge\# /media/File:Menger_sponge_(Level_0-3).jpg) [accessed on 3 September 2021]. When this process is carried out infinitely, the volume of the sponge is null, whereas the surface is 
infinite. Thus, in the $\mathrm{n} t \mathrm{~h}$ iteration the fractal dimension of the object can be determined analytically from Equation (1) as $F_{D}=\ln (20) / \ln (3)=2.727$. The fractal dimension of the Menger sponge is between 2 and 3-that is, it is an object with a dimension greater than a plane, but less than a three-dimensional volume.
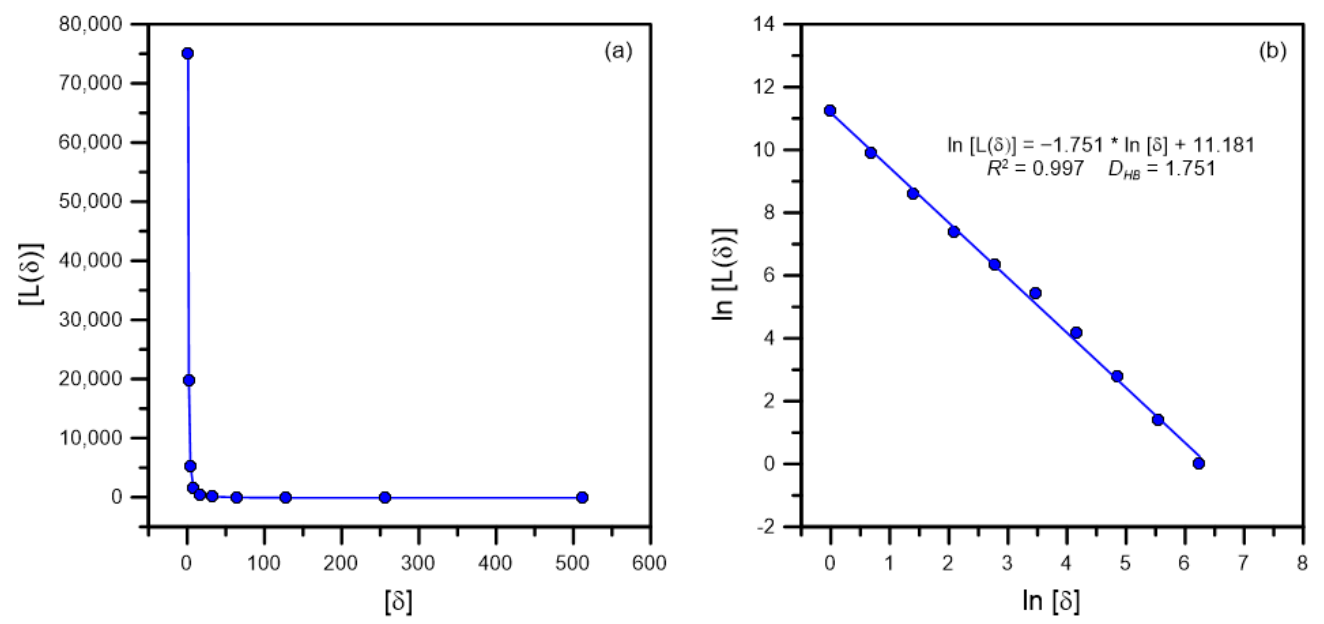

Figure 1. Estimation of the fractal dimension of the third iteration of the Sierpinski carpet. (a) Powerlaw and (b) double logarithmic representations.

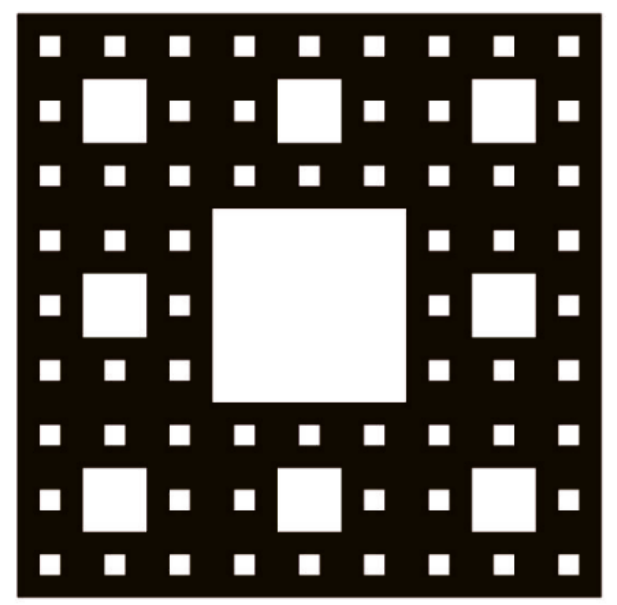

Figure 2. Third iteration of the Sierpinski carpet.

A beam based on this geometry is generated as follows: initially, a beam is constructed by means of an ordered point cloud in Matlab, following the geometry of a prismatic shape. As seen in Figure 3, a total of 70,785 points are obtained from a prism with length 256 and a square section $128 \times 128$, with a resolution $r=4 \times 4$.

Iterative subtractions of material are carried out on the original prismatic beam. This procedure is made by incorporating through-holes of square sections in one of the rectangular faces of the original beam. The objective is to obtain a pattern similar to the one described in the Menger sponge. Finally, a beam based on a fractal geometry is obtained using $N=1,2$ and 3 iterations. In Figure 4 the fractal beam for the second iteration is shown.

The estimation of the fractal dimension of the beams is carried out by means of a box-counting code developed in Matlab using a sequence of box sizes (scales) $\delta=4,8$, $16,32,64$ and 128. Note that whereas the minimum scale is limited by the resolution of the beam $(r=4)$, the maximum is limited by the side of the square section of the beam $(h=a=128)$. 


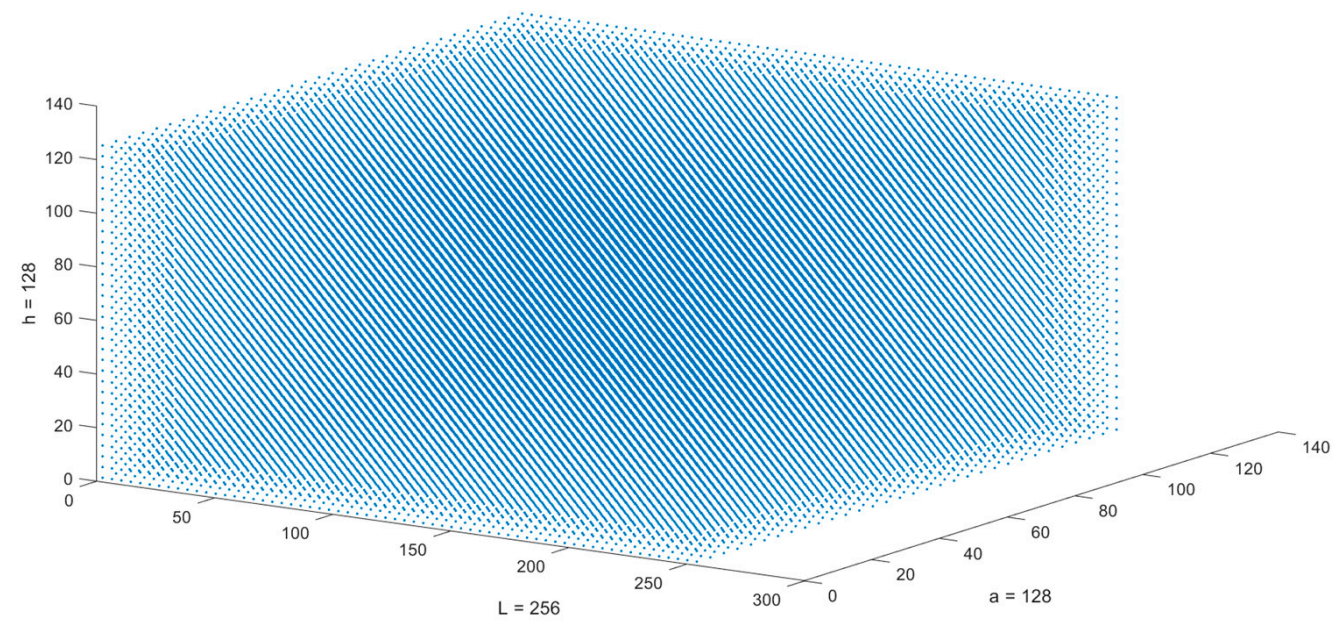

Figure 3. Representation of the original beam. $L=256, h=128, a=128$ and $r=4$.

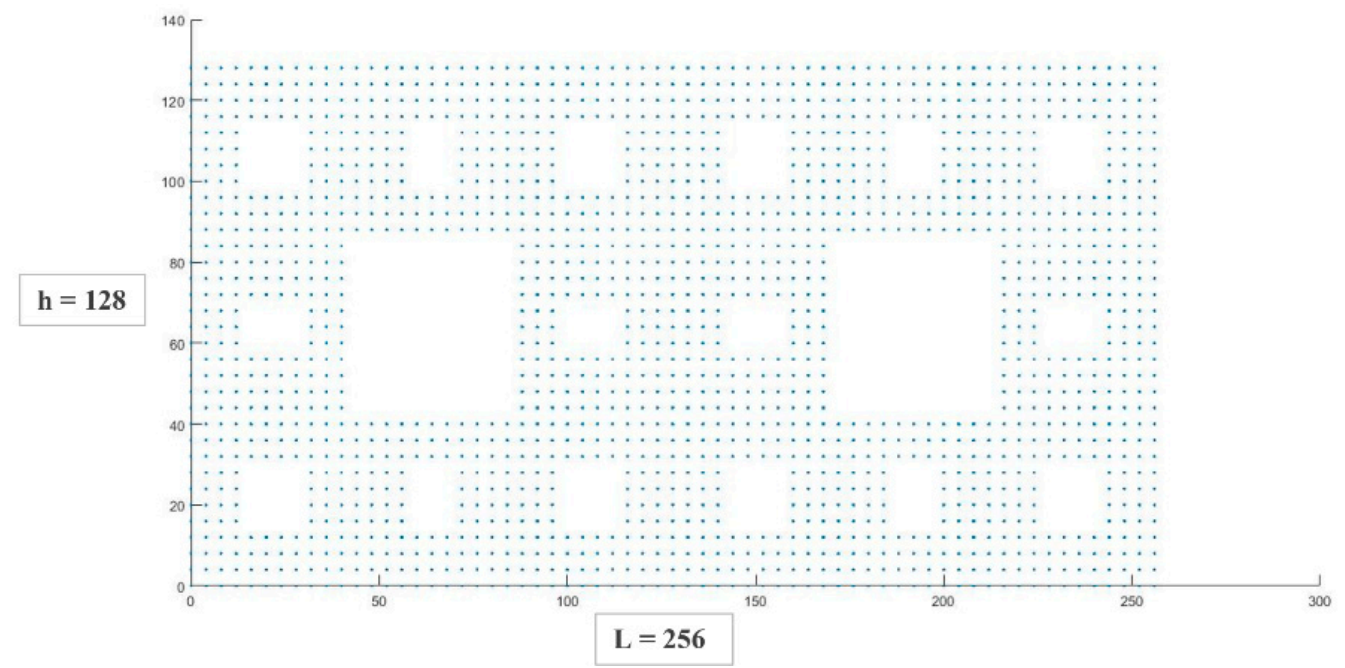

Figure 4. Front view of the beam based on Menger sponge with $N=2$ iterations.

\subsection{Mechanical Resistance Study by Means of Static Simulation}

Inspire software is employed for the mechanical resistance calculation and topology optimization of the beams. For this purpose, an initial prismatic beam with square section is obtained with identical dimensions to those generated in Matlab $(256 \times 128 \times 128)$. Likewise, based on the dimensions of the prismatic beam, an identical beam is modeled with the Menger sponge pattern until iteration $N=3$.

Boundary conditions for resistance simulations were established according to Figure 5. First, a cantilever beam is simulated with a single support on one of its square faces. This square face is understood to be completely embedded in the wall (fixed restriction to movement). The rest of the beam is defined as the design domain, which will be the volume in which the software can lighten material. Second, AISI 304 steel is selected from Inspire's library as material (elastic limit $=200 \mathrm{MPa} E=195,000 \mathrm{MPa} ; \rho=8000 \mathrm{~kg} \mathrm{~m}^{-3}$ ). Third, based on the Michell's beam, a single and vertical force of 500,000 N is established along the entire upper edge of the face opposite to the fixed face. For comparison purposes, boundary conditions are identical in all the simulations. A set of parameters related to the mechanical behavior of the beams are selected as a result: maximum von Mises effort, minimum safety factor and maximum displacement. 


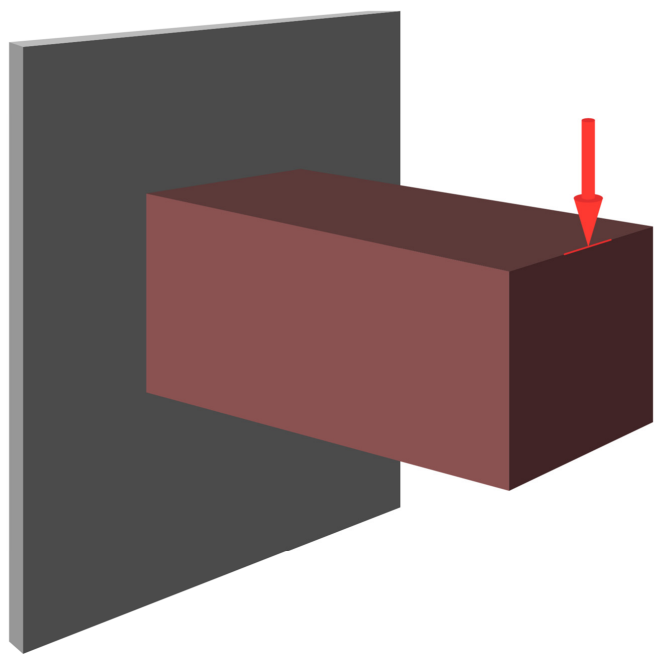

Figure 5. Display of boundary conditions in Inspire software.

\subsection{Topology Optimization}

Topology optimization is also conducted using Inspire software. The optimization function is defined in two different ways: maximizing stiffness, and minimizing mass. In order to obtain realistic solutions, a shape controller is established for obtaining symmetrical pieces with respect to the longitudinal plane of the beams. Although the software allows it, no restrictions on frequencies were considered.

Inspire allows the use of two variables for maximizing stiffness: the percentage of maintained mass and the minimum thickness. In this context, thickness indicates the thinness of the structure supports in the design region of the beam. Therefore, two experiments are established with the objective of maximizing stiffness: OT1, which sets the minimum thickness at $300 \mathrm{~mm}$ and defines a range of maintained mass from $90 \%$ to $10 \%$ at $10 \%$ intervals. Likewise, OT 2 sets the percentage of maintained mass at $50 \%$ and uses the minimum thickness as a variable, which is established in the range from 200 to $500 \mathrm{~mm}$, at $100 \mathrm{~mm}$ intervals. For the experiments whose objective is to minimize the mass, the minimum thickness of the solutions is set $300 \mathrm{~mm}$ and the factor of safety (FOS) is used as a variable, using values from 1 to 3 at 0.25 intervals (OT3). OT4 establishes a constant FOS of 1.2 and uses the minimum thickness as a variable, which is established in the range of 200 to $500 \mathrm{~mm}$, at $100 \mathrm{~mm}$ intervals. In Figure 6, optimization experiments, constants and variables are summarized.

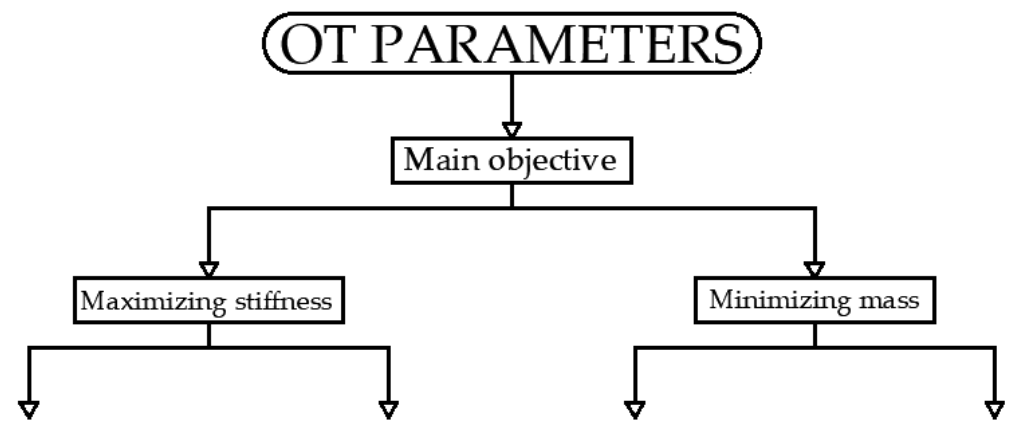

\begin{tabular}{c|cccc} 
Fixed & Thickness & Maintained mass (\%) & Thickness & Factor of safety \\
\hline Variable & Maintained mass (\%) & Thickness & Factor of safety & Thickness \\
& OT1 & OT2 & OT3
\end{tabular}

Figure 6. Decision parameters for topology optimization experiments. 
Finally, the mechanical resistance of the optimized beams is evaluated using the Inspire software according to Section 2.3. Additionally, their fractal dimensions are also estimated. Since the estimation of the fractal dimension is carried out using a box-counting algorithm in Matlab, it is necessary to export the optimized beams from Inspire to a compatible Matlab file. Lightweight solutions are exported using OBJ format. From this format, the $X Y Z$ coordinates of the point cloud comprised by the meshing nodes of the optimized beam can be extracted. This file of coordinates $\mathrm{XYZ}$ is employed as input for determining the fractal dimension by means of the box-counting algorithm in Matlab.

\section{Results and Discussion}

\subsection{Beam Based on the Menger Sponge}

In order to evaluate the fractal generation and static analysis of beams, a prismatic solid beam was first studied. Next, the box-counting algorithm was also used to estimate the fractal dimension of the set of beams based on the Menger sponge. The estimation was carried out for iterations $N=1,2$ and 3 (Table 1). The original solid beam was represented for iteration $N=0$. As expected, the fractal dimension of this solid beam was equal to 3 , which is in agreement with the topological dimension of a 3D volume.

Table 1. Fractal dimension of the set of beams based on the Menger sponge.

\begin{tabular}{ccccccc}
\hline Iteration & $\boldsymbol{F}_{\boldsymbol{D}}$ & Nodes & Mass $(\%)$ & $\sigma_{\text {von Mises }}(\mathbf{M P a})$ & FOS & Dis $_{\max }(\mathbf{m m})$ \\
\hline 0 & 3.000 & 70,785 & $100 \%$ & 308 & 0.65 & 0.96 \\
1 & 2.918 & 52,272 & $74 \%$ & 286 & 0.70 & 1.76 \\
2 & 2.874 & 37,728 & $53 \%$ & 298 & 0.67 & 2.80 \\
3 & 2.657 & 24,317 & $30 \%$ & 409 & 0.49 & 4.66 \\
\hline
\end{tabular}

According to Table 1, the greater the iteration, the closer the estimation to the fractal dimension of the Menger sponge $\left(F_{D}=2.727\right)$. Note that the analytical value cannot be exactly estimated for two reasons. First, the largest iteration reached is $N=3$, which is far from the theoretical infinite iteration which fits with Equation (1). Second, the beam has a prismatic shape and not a cubic geometry, as in the case of the Menger sponge. Therefore, the obtained fractal dimension is accepted as a proper approximation. This also makes it possible to validate the box-counting algorithm used in this work.

Table 1 shows the mechanical resistance parameters obtained from the static simulation. The maximum displacement of the beam $\left(\right.$ Disp $_{\max }$ ) increased with increasing number of iterations. This was expected since the beam was being lightened as the construction of the fractal geometry of the Menger sponge progressed. In order to link the results of the static analysis with the fractal geometry of the beam, fractal dimension versus maximum displacement is depicted in Figure 7. A decreasing relationship between both variables was found, which fit fairly well to a linear fit $\left(R^{2}>0.95\right)$.

\subsection{Beam Based on the Topology Optimization}

According to the methodology described in Section 2.4, we evaluated the potential relationship between the complex geometries obtained from topology optimization and the factors derived from static simulation. Note that identical boundary conditions and design parameters were applied in order to obtain comparable results among optimized beams.

A representation of some optimized beams of the OT1 experiment is depicted in Figure 8 . Notice that the objective in OT1 was to maximize the stiffness by varying the percentage of maintained mass. Lightened solutions with a percentage of maintained mass lower than $20 \%$ were rejected because the beams exhibited discontinuities and they would not be a valid solution for realistic mechanical studies.

Figure 9 shows that the relationship between the maintained mass and the fractal dimension was a convex parabola, which fit to a third-degree polynomial with a goodness of fit $\left(R^{2}\right)$ greater than 0.96 . The fractal dimension reached a maximum value when the percentage of maintained mass was around $70 \%$. Thus, percentages of maintained mass 
greater than $70 \%$ resulted in lower fractal dimensions. Thus, the relationship between the maintained mass and the fractal dimension was not always direct. Lower fractal dimensions were obtained for higher percentages of maintained mass - that is, inefficient geometric configurations for filling the space were obtained when the percentage of maintained mass was greater than $70 \%$. In this case, fractal dimension might serve as an indicator of how the geometry of the beam is distributed in space.

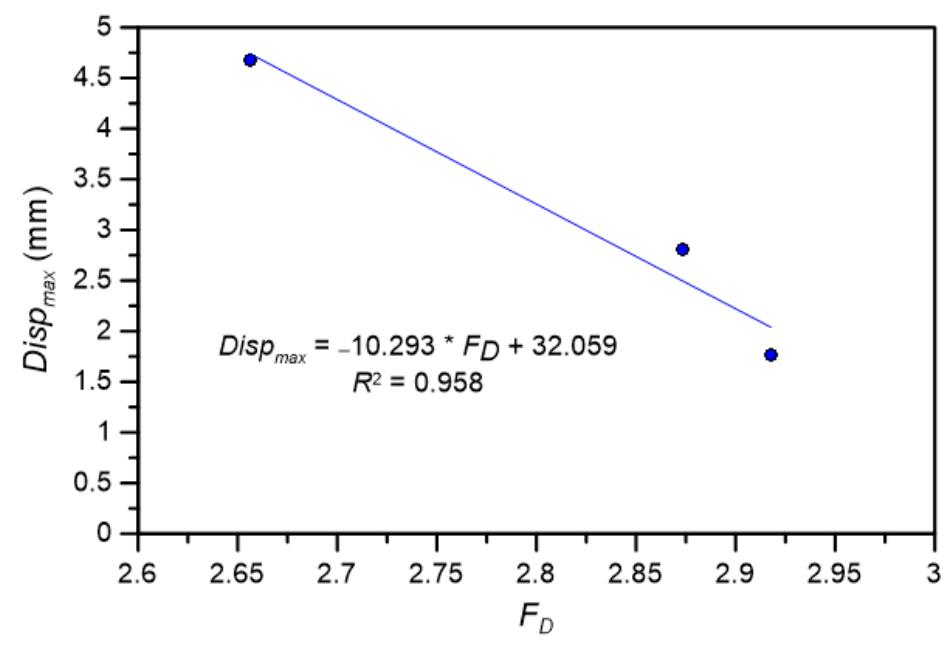

Figure 7. Fractal dimension vs. maximum displacement for different iterations of the Menger sponge.
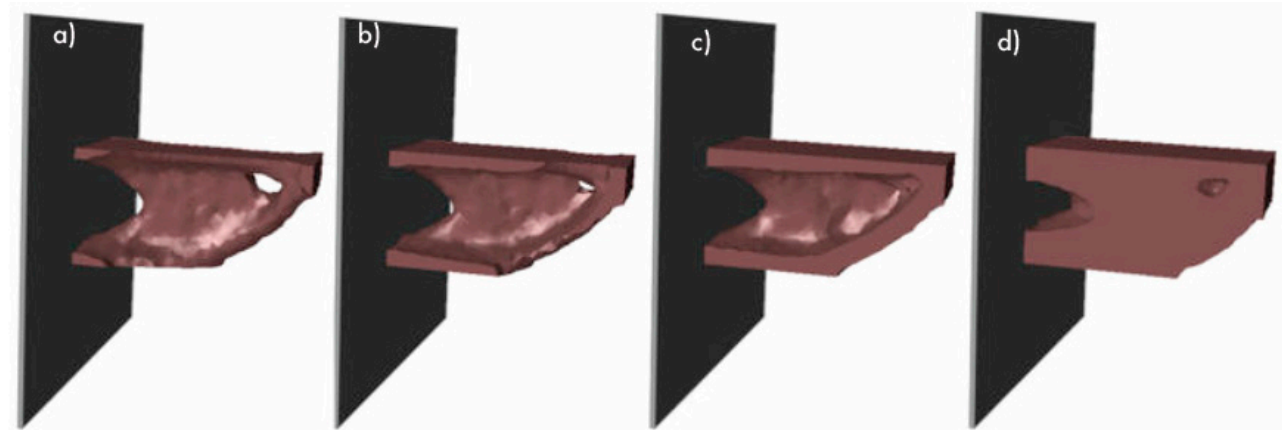

Figure 8. Representation of beams for experiment OT1 maintaining a mass of (a) $30 \%$, (b) $40 \%$, (c) $50 \%$ and (d) $70 \%$.

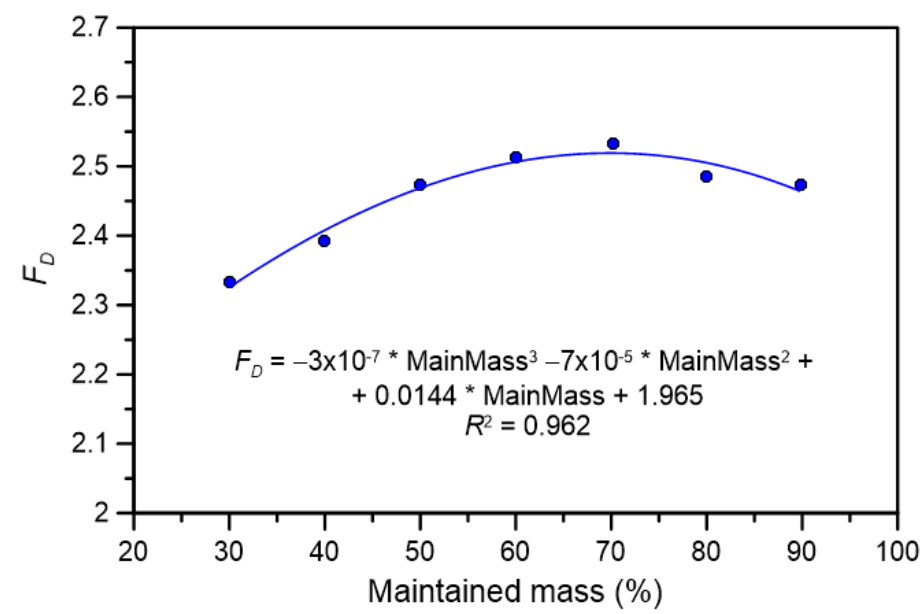

Figure 9. Maintained mass vs. fractal dimension for experiment OT1.

This finding should be analyzed along with Figure 10. In Figure 10, an inversely proportional relationship between the maximum displacement and the percentage of 
maintained mass can be observed. Thus, maintaining a low percentage of mass caused very high displacements in the beam. This decreasing relationship tended towards an asymptotic behavior above a percentage of maintained mass around $70 \%$. Therefore, maintaining masses greater than $70 \%$ led to constant maximum displacements (close to $1 \mathrm{~mm}$ ). This relationship could be fit to a polynomial of degree three with a goodness of $R^{2}>0.99$.

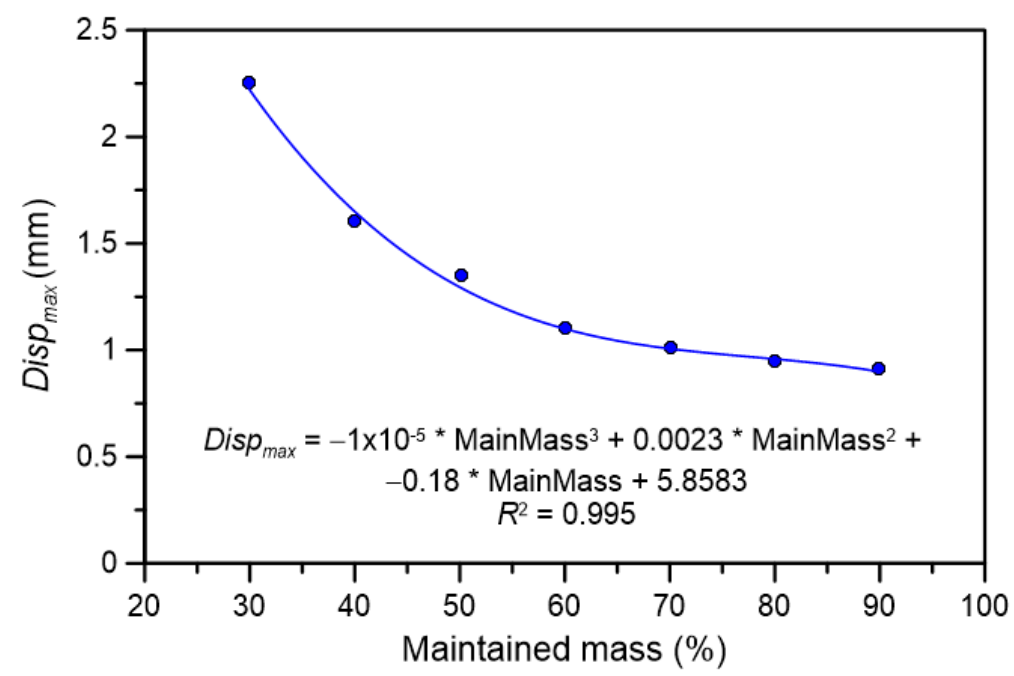

Figure 10. Maintained mass vs. maximum displacement for experiment OT1.

Therefore, to ensure reasonable displacements, percentages of maintained mass in the range $80-90 \%$ are geometrically more inefficient than a maintained mass of $70 \%$. Thus, to ensure acceptable displacements, the fractal dimension can be used as an index of the geometric efficiency of the distribution of a certain mass in space. Von Mises effort and FOS remained practically independent from fractal dimension.

In experiment OT2 the objective was to maximize the stiffness using the thickness as a variable. The maintained mass was fixed at $50 \%$ and the thickness was evaluated from 200 to $500 \mathrm{~mm}$, with increments of $100 \mathrm{~mm}$. The solution for a thickness of $200 \mathrm{~mm}$ was discarded due to discontinuities in the beam. Figure 11 displays the dependence of the fractal dimension on the thickness. For a fixed maintained mass, the influence of thickness on the fractal dimension was negligible because the slope was close to zero in the relationship between both variables. Therefore, fractal dimension cannot be related to mechanical resistance parameters when the thickness of the optimized beams changes.

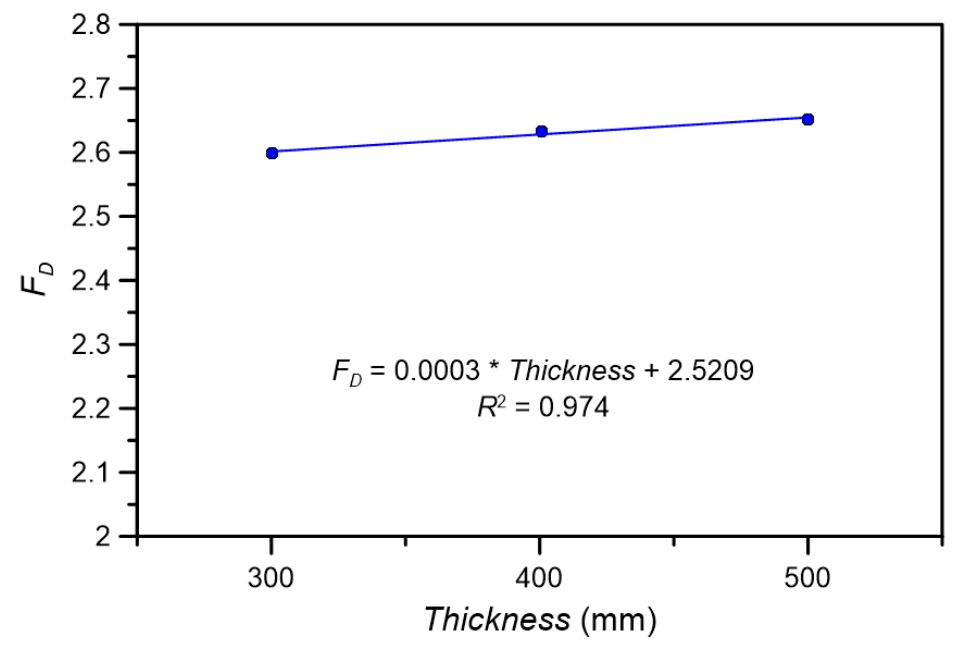

Figure 11. Thickness vs. fractal dimension for experiment OT2. 
The objective of experiment OT3 was to minimize the mass using FOS as the variable (FOS values from 1 to 3 were tested, at 0.25 increments). In Figure 12 some optimized solutions depending on FOS are shown. One of the main findings obtained from experiment OT3 was the relationship between fractal dimension and FOS, which is depicted in Figure 13. The fractal dimension exhibited a positive relationship with FOS, which was linear and could be fit with an $R^{2}>0.94$ (see the filled circles and continuous line in Figure 13). Analogously, the maintained mass followed a positive linear relationship with FOS (see empty circles and dotted line in Figure 13). When the objective was to minimize the mass, higher FOS values were obtained with higher percentages of maintained mass, which is in accordance with the lightened solutions with higher fractal dimension. Therefore, in experiment OT3, the fractal dimension increased simply because the optimized beams had more mass.
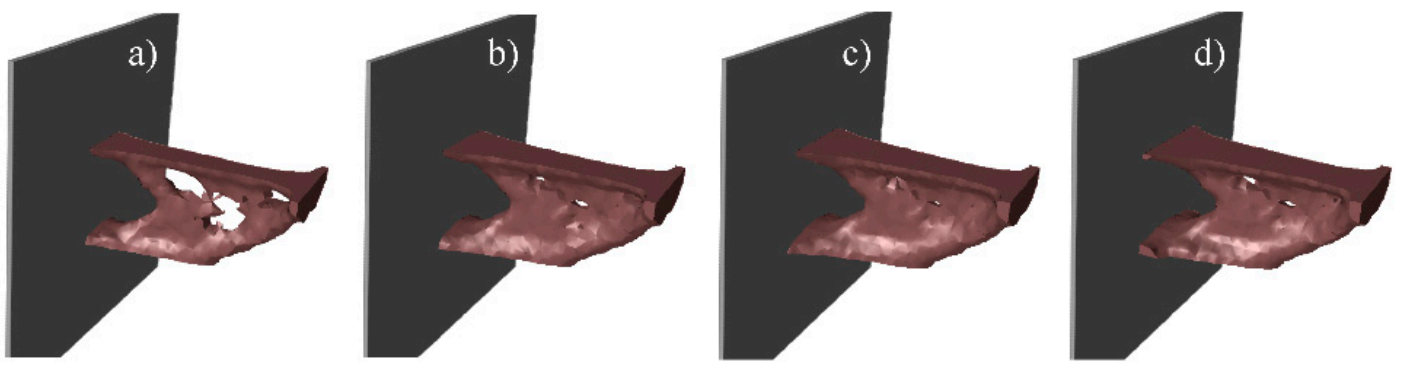

Figure 12. Representation of beams for experiment OT3with an FOS equal to (a) 1, (b) 1.25, (c) 1.75 and (d) 2.

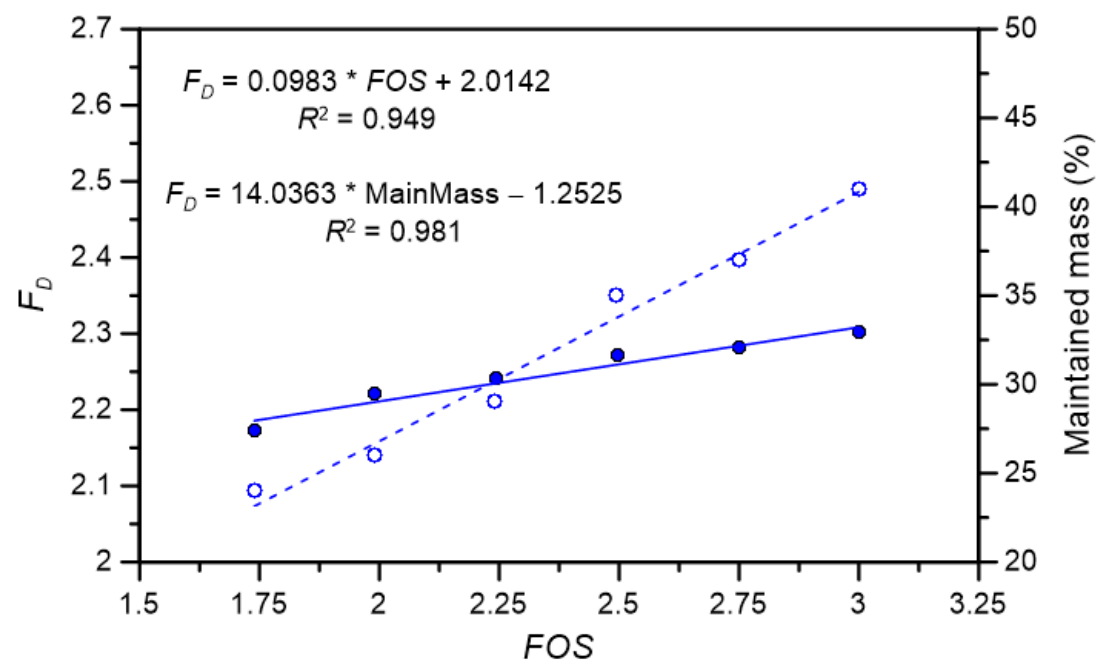

Figure 13. FOS vs. fractal dimension for experiment OT3.

The second main result of experiment OT3 was the inverse relationship between the maximum displacement and the fractal dimension (Figure 14). This decreasing relationship could be adjusted to a polynomial of degree 2 , with a goodness of fit $R^{2}>0.97$. In this case, the relationship did not show an asymptotic region as it did in OT1. The fractal dimension increased along with the maintained mass, and thus the fractal dimension cannot be used as a parameter for assessing the geometric efficiency of the solutions when the objective is to minimize the mass.

In experiment OT4, the objective was to minimize the mass using the thickness as a variable. Thickness was evaluated with values from 200 to $500 \mathrm{~mm}$, with increments of $100 \mathrm{~mm}$. As in OT2, the results indicate that the fractal dimension is not related to mechanical resistance parameters when the thickness of the optimized beams changes. 


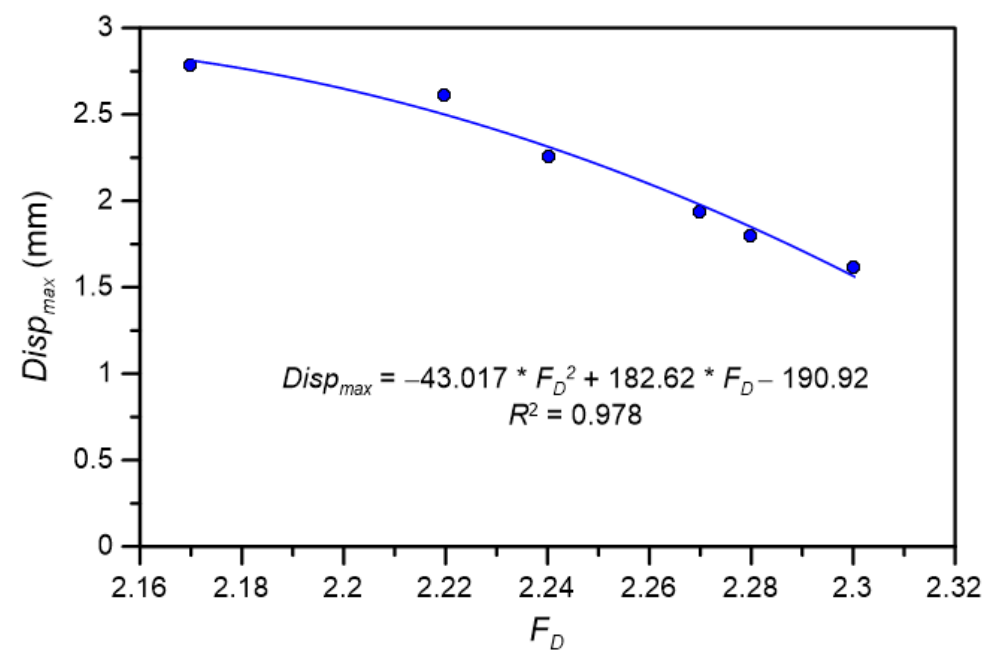

Figure 14. Fractal dimension vs. maximum displacement for experiment OT3.

\section{Conclusions}

Topology optimization focuses on the percentage of maintained mass to ensure compliance with a set of objectives and restrictions due to the mechanical stress to which a piece is subjected. If restrictions are met, the optimum lightweight solution maintains the lowest percentage of mass, which is related to a reduction of the material cost. In these cases, the material is efficiently distributed throughout the design space in order to sustain and resist mechanical stresses. However, the parameter percentage of maintained mass does not provide information on the efficiency of the material in distributing itself through the geometric space. Furthermore, it is not possible to discern which optimized piece best distributes its mass when several solutions with similar maintained mass are obtained.

In this work, fractal dimension was first tested as a parameter in a topology optimization framework. It was evaluated for both characterizing complex geometries in structural pieces and providing complementary information to the traditional maintained mass. A cantilever beam with a single force applied at the end was employed as an example in this work. Initially, a lightening based on a Menger sponge was carried out on the beam to test the method for estimating the fractal dimension, as well as the procedure for calculating the mechanical parameters of the study (von Mises stress, factor of safety, and maximum displacement).

Regarding topology optimization, some findings were remarkable. When the objective was to maximize the stiffness (OT1), a relationship among the geometry, fractal dimension and maximum displacement was observed. The fractal dimension increased with the percentage of maintained mass until around $70 \%$, at which point the fractal dimension began to decrease. This is because fractal dimension not only depends on the amount of material but on how that material is distributed in space. Therefore, the percentage of maintained mass for which the fractal dimension reached a maximum value was found. This maximum was associated with an asymptotic behavior in the maximum displacement. Thus, the results suggest that when the objective of the optimization is to maximize stiffness, fractal dimension is a useful index for determining the optimum percentage of maintained mass, which is also related to stable maximum displacement. However, when the objective is to minimize the mass (OT3), the relationship between fractal dimension and maximum displacement was a decreasing curve. Variations in the thickness of the topology optimization solutions influenced neither the fractal dimension nor the maximum displacement (OT2 and OT4).

In summary, our results indicate that when the objective of the topology optimization is to maximize the stiffness, fractal dimension is related to the amount of maintained mass as well as the geometric distribution of the material. Therefore, we suggest that fractal dimension is a useful parameter for describing the geometric efficiency of the lightened beam. By contrast, when minimizing mass is used as a design objective, our results indicate 
that fractal dimension is only a parameter linked to the amount of mass held in the piece. Note that the main limitations of this study are conditioned by the typology of beam chosen (a cantilever beam). In future work, it might be interesting to develop similar studies on other types of beams (double-embedded, bi-supported, etc.), with different loads (point, uniformly distributed, etc.), as well as different sections and geometries. Overall, the results of this study suggest that it could be promising to add the fractal dimension as an additional parameter for making decisions in topology optimization software.

Author Contributions: Conceptualization, P.P.-D. and L.R.-P.; methodology, P.P.-D. and G.P.-G.; software, P.P.-D. and G.P.-G.; validation, P.P.-D., G.P.-G. and L.R.-P.; formal analysis, P.P.-D. and A.R.-C.; investigation, P.P.-D., A.R.-C. and L.R.-P.; resources, A.R.-C. and L.R.-P.; data curation, P.P.-D. and G.P.-G.; writing-original draft preparation, P.P.-D. and G.P.-G.; writing-review and editing, P.P.-D., A.R.-C. and L.R.-P.; visualization, P.P.-D. and G.P.-G.; supervision, P.P.-D. and L.R.-P.; project administration, P.P.-D. and L.R.-P. All authors have read and agreed to the published version of the manuscript.

Funding: This work was supported for publication by the "Conselleria de Innovación, Universidades, Ciencia y Sociedad Digital" of the "Generalitat Valenciana". The authors would like to thank also to the INGEGRAF association.

Institutional Review Board Statement: Not applicable.

Informed Consent Statement: Not applicable.

Data Availability Statement: The data presented in this study are available on request from the corresponding author.

Conflicts of Interest: The authors declare no conflict of interest.

\section{References}

1. Mandelbrot, B. How Long Is the Coast of Britain? Statistical self-similarity and fractional dimension. Science 1967, 156, 636-638. [CrossRef]

2. González, V.A.; Guerrero, C. Fractales: Fundamentos y aplicaciones, Parte I: Concepción geométrica en la ciencia e Ingeniería. Ingenierías IV 2001, 10, 53-59.

3. Agrawal, Y.; Ananthasuresh, G.K. Towards optimal heterogeneity in lattice structures. Struct. Multidiscip. Optim. 2021, 64, 2489-2512. [CrossRef]

4. Díaz Lantada, A.; Lafont Morgado, P.; Ortego García, P.; Muñoz-Guijosa, J.M.; Sanz, J.M.; Otero, J.E.; Tanarro EC de la Guerra Ochoa, E. Diseño y fabricación rápida de geometrías biomiméticas fractales en materiales biocompatibles para aplicaciones en ingeniería de tejidos. In Proceedings of the XIX Congreso Nacional de Ingeniería Mecánica, Castellón de la Plana, Spain, 14-16 November 2012

5. Qin, Z.; Compton, B.G.; Lewis, J.A.; Buehler, M.J. Structural optimization of 3D-printed synthetic spider webs for high strength Nat. Commun. 2015, 6, 7038. [CrossRef]

6. Zhang, H.-K.; Wu, W.-J.; Kang, Z.; Feng, X.-Q. Topology optimization method for the design of bioinspired self-similar hierarchical microstructures. Comput. Method. Appl. Mech. Eng. 2020, 372, 113399. [CrossRef]

7. Skelton, R.E.; de Oliveira, M.C. Tensegrity Systems; Springer: New York, NY, USA, 2010.

8. Gonzalez, M.; Jelisavcic, N.; Moral, R.J.; Sahoo, D.; Dulikravich, G.S.; Martin, T.J. Multi-objective design optimization of topology and performance of branching networks of cooling passages. Int. J. Therm. Sci. 2007, 46, 1191-1202. [CrossRef]

9. Ozguc, S.; Pan, L.; Weibel, J.A. Topology optimization of microchannel heat sinks using a homogenization approach. Int. J. Heat. Mass Transf. 2021, 169, 120896. [CrossRef]

10. Tian, Y.; Liu, X.; Xu, Q.; Luo, Q.; Zheng, H.; Song, C.; Zhu, Z.; Gao, K.; Dang, C.; Wang, H.; et al. Bionic topology optimization of fins for rapid latent heat thermal energy storage. Appl. Therm. Eng. 2021, 194, 117104. [CrossRef]

11. Xiao, B.; Wang, W.; Fan, J.; Chen, H.; Hu, X.; Zhao, D.; Zhang, X.; Ren, W.E.N. Optimization of the fractal-like architecture of porous fibrous materials related to permeability, diffusivity and thermal conductivity. Fractals 2017, 25, 1750030. [CrossRef]

12. Bendsøe, M.P.; Sigmund, O. Topology Optimization-Theory, Methods, and Applications, 2nd ed.; Springer: Berlin/Heidelberg, Germany, 2004.

13. Millán Páramo, C.; Begambre Carrillo, O. Solución de problemas de optimización topológica empleando el Algoritmo Simulated Annealing Modificado. Rev. Int. Metodos Numer. Calc. Diseno Ing. 2016, 32, 65-69. [CrossRef]

14. Michell, A.G.M. The limits of economy of material in frame-structures. Lond. Edinb. Dubl. Phil. Mag. 1904, 8, 589-597. [CrossRef]

15. Sotola, M.; Marsalek, P.; Rybansky, D.; Fusek, M.; Gabriel, D. Sensitivity analysis of key formulations of topology optimization on an example of cantilever bending beam. Symmetry 2021, 13, 712. [CrossRef]

16. Sigmund, O.; Maute, K. Topology optimization approaches. Struct. Multidiscip. Optim. 2013, 48, 1031-1055. [CrossRef] 
17. Rozvany, G.I.N. Grillages of maximum strength and maximum stiffness. Int. J. Mech. Sci. 1972, 14, 651-666. [CrossRef]

18. Rozvany, G.I.N. Optimal load transmission by flexure. Comput. Method. Appl. Mech. Eng. 1972, 1, 253-263. [CrossRef]

19. Skelton, R.E.; de Oliveira, M.C. Optimal complexity of deployable compressive structures. J. Frankl. Inst. 2010, 347, 228-256. [CrossRef]

20. Skelton, R.E.; de Oliveira, M.C. Optimal tensegrity structures in bending: The discrete Michell truss. J. Frankl. Inst. 2010, 347, 257-283. [CrossRef]

21. Skelton, R.E.; Fraternali, F.; Carpentieri, G.; Micheletti, A. Minimum mass design of tensegrity bridges with parametric architecture and multiscale complexity. Mech. Res. Commun. 2014, 58, 124-132. [CrossRef]

22. Nigro Lopes, H.; Candeloro Cunha, D.; Pavanello, R.; Mahfoud, J. Numerical and experimental investigation on topology optimization of an elongated dynamic system. Mech. Syst. Sig. Process. 2022, 165, 108356. [CrossRef]

23. Li, H.; Kondoh, T.; Jolivet, P.; Furuta, K.; Yamada, T.; Zhu, B.; Izui, K.; Nishiwaki, S. Three-dimensional topology optimization of a fluid-structure system using body-fitted mesh adaption based on the level-set method. Appl. Math. Model. 2022, 101, 276-308. [CrossRef]

24. Feder, J. Fractals; Plenum Press: New York, NY, USA, 1988.

25. Lopez, R.; Betrouni, N. Fractal and multifractal analysis: A review. Med. Image Anal. 2009, 13, 634-649. [CrossRef] [PubMed] 